\title{
Simulating Twistronics without a Twist
}

\author{
Tymoteusz Salamon $\odot,{ }^{1}$ Alessio Celiø, ${ }^{2}$ Ravindra W. Chhajlany $\odot,{ }^{3}$ Irénée Frérot $\odot,{ }^{1,4}$ Maciej Lewenstein, ${ }^{1,5}$ \\ Leticia Tarruellø, ${ }^{1}$ and Debraj Rakshit $\odot^{1,4}$ \\ ${ }^{1}$ ICFO-Institut de Ciencies Fotoniques, The Barcelona Institute of Science and Technology, \\ 08860 Castelldefels (Barcelona), Spain \\ ${ }^{2}$ Departament de Física, Universitat Autònoma de Barcelona, 08193 Bellaterra, Spain \\ ${ }^{3}$ Faculty of Physics, Adam Mickiewicz University, 61614 Poznan, Poland \\ ${ }^{4}$ Max-Planck-Institut für Quantenoptik, D-85748 Garching, Germany \\ ${ }^{5}$ Institució Catalana de Recerca i Estudis Avançats (ICREA), \\ Passeig Lluis Companys 23, ES-08010 Barcelona, Spain
}

(Received 8 February 2020; accepted 22 June 2020; published 14 July 2020)

\begin{abstract}
Rotational misalignment or twisting of two monolayers of graphene strongly influences its electronic properties. Structurally, twisting leads to large periodic supercell structures, which in turn can support intriguing strongly correlated behavior. Here, we propose a highly tunable scheme to synthetically emulate twisted bilayer systems with ultracold atoms trapped in an optical lattice. In our scheme, neither a physical bilayer nor twist is directly realized. Instead, two synthetic layers are produced exploiting coherently coupled internal atomic states, and a supercell structure is generated via a spatially dependent Raman coupling. To illustrate this concept, we focus on a synthetic square bilayer lattice and show that it leads to tunable quasiflatbands and Dirac cone spectra under certain magic supercell periodicities. The appearance of these features are explained using a perturbative analysis. Our proposal can be implemented using available state-of-the-art experimental techniques, and opens the route toward the controlled study of strongly correlated flatband accompanied by hybridization physics akin to magic angle bilayer graphene in cold atom quantum simulators.
\end{abstract}

DOI: 10.1103/PhysRevLett.125.030504

Novel routes to band engineering in lattice systems often lead to fundamental questions and new material functionalities. Different schemes of stacking two-dimensional layers have emerged as a fruitful way of modifying material properties through the design of supercell structures and opened the field of so-called Van der Waals materials [1]. In particular, twisted bilayer graphene (TWBLG) has emerged as a tunable experimental platform hosting flatband physics and strongly correlated phenomena, such as possibly unconventional superconductivity, magnetism, and other exotic phases [2-5]. This has inspired much theoretical debate around the origin of the electronic properties of TWBLG [6-21].

The interesting correlated phenomenology is apparently related to moiré patterns around small twist angles, the socalled magic angles, which lead to band flattening or effective mass reduction already at the single-particle level

Published by the American Physical Society under the terms of the Creative Commons Attribution 4.0 International license. Further distribution of this work must maintain attribution to the author(s) and the published article's title, journal citation, and DOI. Open access publication funded by the Max Planck Society.
[22-26]. The geometrical moiré patterns physically induce spatially varying interlayer couplings that are behind the strong modification of the band structure. As in artificial graphene systems [27], emulating this physics beyond materials research may allow identifying key minimal ingredients that give rise to the phenomenology of TWBLG while also providing additional microscopic control. Photonic systems are particularly suited for exploring this physics at the single-particle level. Very recently, single-particle transport in tunable photonic moiré lattices has been experimentally studied [28], where two dimensional localization of light and localization-delocalization have been experimentally demonstrated. Ultracold atoms in optical lattices $[29,30]$ are also the most promising platform for experimentally exploring the corresponding emerging manybody phenomena. The experimental realization of artificial graphene geometries [31,32], lattice geometries displaying flatbands like Kagome [33] and Lieb [34,35], or quasicrystal structures [36-38], provides the building blocks for such exploration.

One obvious approach for studying twisted bilayer graphene physics with ultracold atoms is to directly implement twisted bilayers using two intertwined optical lattices, as recently proposed in Ref. [39]. Schemes for 
simulating other bilayer heterostructures have also been put forward [40]. This direct strategy poses significant experimental challenges, as it is difficult to stabilize the two layers at relative small angles and simultaneously achieve a sufficiently large lattice containing several supercells. Here, we propose an alternative scheme that builds on the concept of synthetic dimensions, i.e., reinterpreting the coherent Raman coupling between spin states of an atom as controllable tunneling along an artificial extra dimension [41-43]. For this, we note that the key effect of physical twisting is to induce spatially modulated interlayer tunnelings across the lattice. In our scheme, the modulated interlayer tunneling patterns are proposed to be directly imprinted on the lattice via spatial control of the Raman couplings, thus, amounting to twisting the system without a physical twist. This leads to the creation of supercells with controllable sizes and shapes. By adjusting the strength, phase, and spatial periodicity of $\Omega$, we show that an exemplary bilayer square lattice system supports a broad range of band structures. In particular, magic values of the periodicity result in the appearance of (quasi) flatbands as well as Dirac cone spectra. Although we focus in our illustrative example on a particular spatial modulation, general interlayer coupling patterns can be experimentally induced including quasiperiodic or moiré-like patterns. Our proposal can be realized with fermionic two-electron atoms, such as strontium or ytterbium, using available experimental techniques.

Concept.-We consider a two-dimensional Fermi gas with four internal states, indicated here generically as spin states $\{m, \sigma\}= \pm 1 / 2$. The system is loaded into a spinindependent square optical lattice of lattice spacing $d$, which lies in the $x-y$ plane and is characterized by a real tunneling amplitude $t$. We select two states to play the role of the electron spin $\sigma=\uparrow$, and the other two of electron spin $\sigma=\downarrow$. In addition, spin states corresponding to the same $\sigma$ are coupled in pairs. We label them by the index $m$, and make them play the role of a synthetic layer dimension. Since $m= \pm 1 / 2$, we obtain a bilayer structure of synthetic layer tunneling given by the coherent coupling. In order to obtain a lattice geometry with a tunable supercell, we choose the amplitude of the synthetic layer tunneling to be spatially modulated according to $\Omega(x, y)=\Omega_{0}\left\{1-\alpha\left[1+\cos \left(2 \pi x / l_{x}\right) \cos \left(2 \pi y / l_{y}\right)\right]\right\}$. Here, $l_{x}\left(l_{y}\right)$ is its periodicity along the $x(y)$ axis. The synthetic tunneling also induces a Peierls phase $\boldsymbol{\gamma} \cdot \mathbf{r}$, where $\boldsymbol{\gamma}=\gamma(\hat{x}+\hat{y})$ and $\boldsymbol{r}=x \hat{x}+y \hat{y}$. This mimics the effect of a magnetic flux that pierces the system perpendicularly to the synthetic layer dimension [42]. As depicted in Fig. 1(a), the complete scheme represents a synthetic spinful bilayer structure subjected to a magnetic field, denoted as $\Theta\left(l_{x}, l_{y}\right)$.

The Hamiltonian of the system is given by

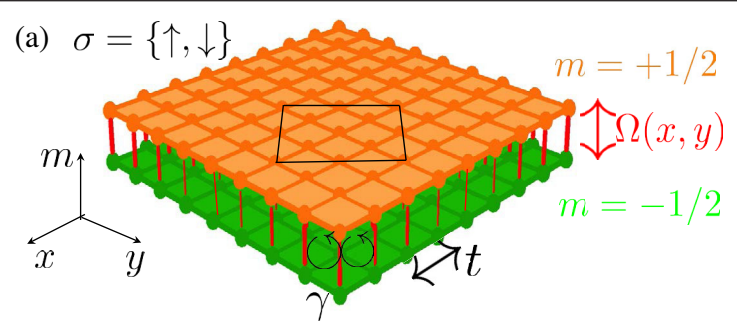

(b)
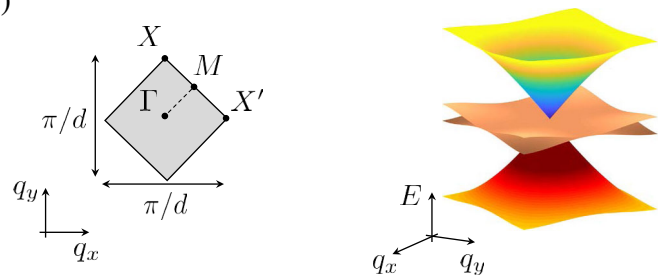

(c)
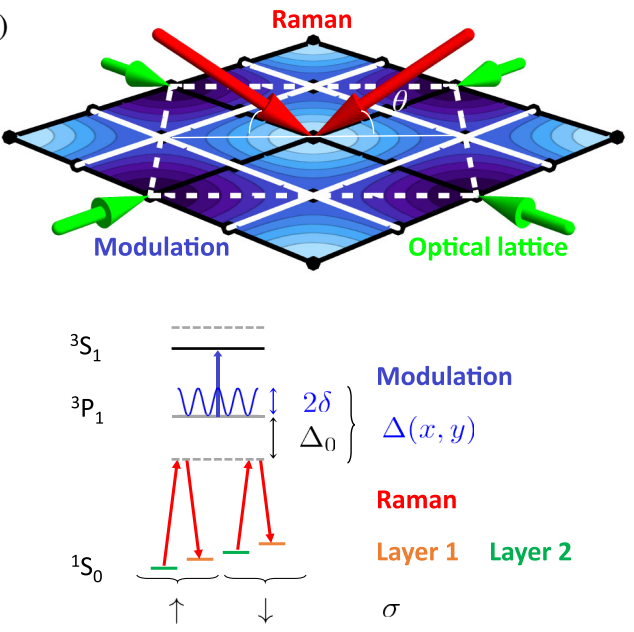

FIG. 1. Synthetic bilayer structure with a supercell. (a) Realspace potential of the synthetic bilayer. Each plane corresponds to one spin state $m= \pm 1 / 2$ (orange, green), which experiences a square lattice potential (tunneling $t$ ) and is connected to the other layer by a spatially dependent and complex coupling $\Omega(x, y)$ (vertical red lines of variable width). A top view of the lattice indicating the unit cell of the system containing $2 \times 8$ sites for $l_{x}=l_{y}=4 d$ is shown (black line). (b) Sketch of the first Brillouin zone, indicating the position of the high-symmetry points, and three dimensional view of the energy spectrum in the vicinity of $E=-\Omega_{0}(1-\alpha)$ for $\Omega_{0} \alpha / h=20 t$ with $\alpha=0.2$ and $\gamma=0$. It has two quasiflatbands intersecting a Dirac point. Note that a simple square lattice supports neither flatbands nor Dirac cones. (c) Proposed experimental realization. Top: Two retroreflected optical lattice beams (green) create the square lattice. Two Raman beams of opening angle $\theta$ (red) produce complex synthetic tunneling between the two layers. One "modulation laser" with a spatially varying intensity distribution (blue) modulates the amplitude of the Raman coupling. Bottom: laser beams involved in the synthetic bilayer coupling scheme. The single-photon detuning of the Raman beams (red arrows) is spatially modulated with respect to its initial value $\Delta_{0} / 2 \pi \sim$ $75 \mathrm{MHz}$ using a laser beam blue detuned with respect to the ${ }^{3} P_{1} \rightarrow{ }^{3} S_{1}$ transition (blue arrow). It produces a light shift of maximal amplitude $2 \delta / 2 \pi \sim 30 \mathrm{MHz}$. 


$$
H=H_{\text {in }}+H_{\text {inter }}=-t \sum_{\mathbf{r}, m, \sigma}\left[a_{m, \sigma}^{\dagger}(\mathbf{r}+d \hat{x})+a_{m, \sigma}^{\dagger}(\mathbf{r}+d \hat{y})+\Omega(\mathbf{r}) \exp (-i \gamma \cdot \mathbf{r}) a_{m+1, \sigma}^{\dagger}(\mathbf{r})\right] a_{m, \sigma}(\mathbf{r})+\text { H.c. },
$$

where we distinguish the in-layer and the interlayer tunnelings.

To diagonalize it, we combine a gauge transformation and a Fourier transform such that $a_{m, \sigma}(\mathbf{r})=$ $\sum_{\mathbf{q}} \exp [i(\mathbf{q} \cdot \mathbf{r}+m \boldsymbol{\gamma} \cdot \mathbf{r})] a_{m, \sigma}(\mathbf{q})$. Here, $\mathbf{q}$ is the momentum conjugated to $\mathbf{r}$. The Hamiltonian can then be rewritten as $H=\sum_{\mathbf{q}} H_{\mathbf{q}}$, where the dimension of $H_{\mathbf{q}}$ is set by the spatial periodicity of the synthetic tunneling. Figure 1(b) sketches the Brillouin zone of the bilayer system and a three-dimensional view of its energy spectrum for $l_{x}=l_{y}=4 d$, corresponding to $\Theta(4,4)$, for $\gamma=0$. In the vicinity of $E= \pm \Omega_{0}(1-\alpha)$, it features two quasiflatbands and a Dirac point touching them [only one of them is represented in Fig. 1(b)]. This band structure is reminiscent of that of magic angle twisted bilayer graphene.

Experimental proposal.-For specificity, we focus on the realization of this scheme employing a subset of four states out of the large nuclear spin manifold $I=9 / 2$ of ${ }^{87} \mathrm{Sr}$. Note, however, that our proposal is directly transposable to ${ }^{173} \mathrm{Yb}(I=5 / 2)$. Thanks to the $S U(N)$ invariant interactions characteristic of two-electron systems, collisional redistribution of the atoms among the different states is inhibited. We select two of them to play the role of the electron spin $\sigma=\uparrow$, and the other two of spin $\sigma=\downarrow$. All are subjected to a two-dimensional spin-independent optical lattice potential, created by two counter-propagating lattice beams. We choose $\lambda_{L}=813 \mathrm{~nm}$, which is commonly used because it corresponds to the magic wavelength of the clock transition ${ }^{1} S_{0} \rightarrow{ }^{1} P_{0}$. We set a lattice depth $8 E_{L}$, which yields $t / h=107 \mathrm{~Hz}$. Here, $E_{L}=$ $\hbar^{2} k_{L}^{2} / 2 m$ is the lattice recoil energy, $k_{L}=2 \pi / \lambda_{L}$, and $d=\lambda_{L} / 2$.

To create the synthetic layer tunneling, we exploit twophoton Raman transitions between spins $m= \pm 1 / 2$ [44]. We employ a pair of Raman beams of wavelength $\lambda_{R}=$ $689 \mathrm{~nm}$ near resonant to the intercombination transition ${ }^{1} S_{0} \rightarrow{ }^{3} P_{1}$, which produces a coupling of amplitude $\Omega_{0}=\Omega_{1} \Omega_{2} / \Delta_{0}$. Here, $\Omega_{1}$ and $\Omega_{2}$ are the individual coupling amplitudes of the Raman lasers and $\Delta_{0}$ the single-photon detuning. The Raman beams propagate in a plane perpendicular to the lattice potential, are aligned along its diagonal, and form an angle $\theta$ with the lattice plane [see Fig. 1(c)]. This yields an in-plane momentum transfer per beam $k_{R}= \pm 2 \pi \cos \theta / \lambda_{R}$, with projections $k_{R} / \sqrt{2}$ along the lattice axes. Therefore, the phase of the synthetic tunneling is $\gamma \cdot \mathbf{r}=\gamma(x \hat{x}+y \hat{y})$, with $\gamma= \pm 2 \pi \cos \theta \lambda_{L} /\left(\sqrt{2} \lambda_{R}\right)$. The sign is determined by the relative detuning of the Raman lasers. Experimentally, the simplest choice is to use counterpropagating Raman beams $\left(\theta=0^{\circ}\right)$, which yields $\gamma=0.8(\bmod 2 \pi)$. However, other magnetic fluxes can be easily realized by adjusting the value of $\theta$.

To implement a periodic modulation of the Raman coupling amplitude on the scale of several lattice sites, which is the key ingredient of our scheme, we propose to exploit a periodic potential created by a laser close-detuned from the excited state to excited state transition ${ }^{3} P_{1} \rightarrow{ }^{3} S_{1}$ (corresponding to $688 \mathrm{~nm}$ [46]). This results in a large light shift of the ${ }^{3} P_{1}$ excited state of amplitude $\delta$, leading to a detuning of the Raman beams $\Delta(x, y)=\Delta_{0}+\delta\left[1+\cos \left(2 \pi x / l_{x}\right) \cos \left(2 \pi y / l_{y}\right)\right]$. Its effect is to modulate the Raman coupling amplitude $\Omega(x, y) \simeq \Omega_{0}\left[(1-\alpha)-\alpha \cos \left(2 \pi x / l_{x}\right) \cos \left(2 \pi y / l_{y}\right)\right]$, with $\alpha=\delta / \Delta_{0} \sim 0.2$ for realistic experimental parameters, see Fig. 1(c) [46,47]. Therefore, we name it modulation laser. Band structures analogous to the one depicted in Fig. 1(b) are obtained for large values of $\alpha \Omega_{0} / h \gtrsim 20 t=10.7 \mathrm{kHz}$ and spatial periodicities of the Raman coupling of several lattice sites [48]. The necessary patterns can be projected by combining a spatial light modulator and an optical system of moderate optical resolution, ensuring a large flexibility.

Magic configurations. - The emerging band structures are sensitive to the spatial modulation and the strength of the laser coupling. Typically, a system with weak Raman coupling $\left(\Omega_{0} \alpha / h \lesssim t\right)$ hosts a large number of extended hybridized bands. Enhanced coupling strength $\left(\Omega_{0} \alpha / h \approx 10 t\right)$ tends to foster band narrowing. Remarkably, there exist magic configurations of our considered bilayer square lattice for which special band structures emerge-quasiflatbands surrounded by dispersive Dirac cone spectra with controllable Dirac velocities. We quantify flatness $F$ of a band by the ratio between its width and the dispersion of neighboring bands (cf. [26]). This ratio and the emergence of quasiflatbands can be understood and calculated in perturbation theory (see Supplemental Material [49]).

The configuration $\Theta(4,4)$ corresponds to the smallest bilayer supercell, consisting of $(2 \times 8)$ sites, supporting this band structure. Figure 1(b) shows the resulting spin degenerate bands around $E / t=-\Omega_{0}(1-\alpha)$ for an exemplary case with strong Raman coupling $\Omega_{0} \alpha / h=20 t, \alpha=$ 0.2 and vanishing flux $\gamma=0$. Increasing the flux to the simplest experimentally attainable value of $\gamma=0.8$ shares many of the features of the fluxless case. Narrow groups of bands, that are well separated from each other, are formed for sufficiently large $\Omega_{0} \alpha$ at the energies $\pm \Omega_{0}, \pm \Omega_{0}(1-\alpha)$, and $\pm \Omega_{0}(1-2 \alpha)$ (see Fig. S3 in [49]). The full spectrum of the system is symmetric around $E=0$, so we only discuss 


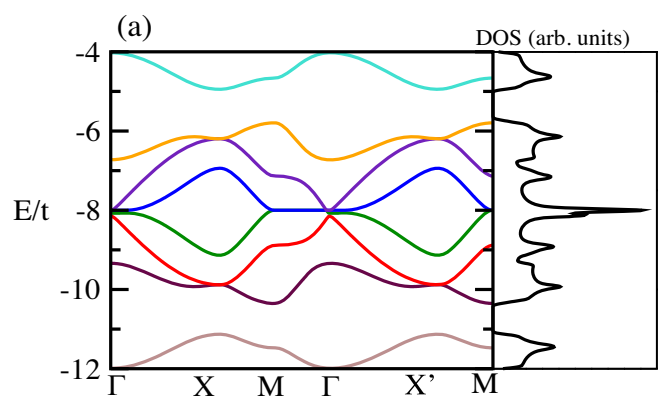

(b)

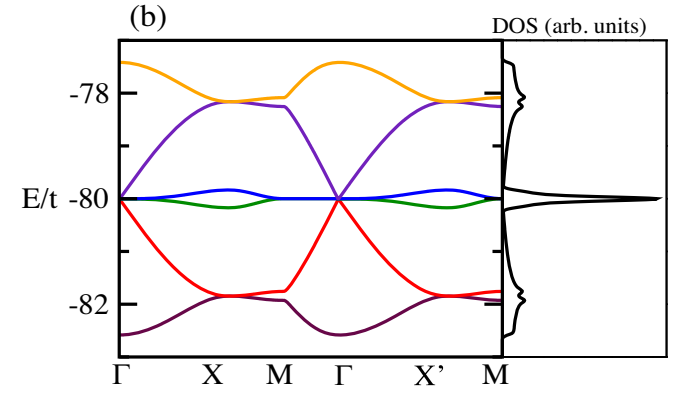

FIG. 2. Magic configuration band structure and DOS. Band structures around energy $-\Omega_{0}(1-\alpha)$ and DOS (in arbitrary units) corresponding to $\Theta(4,4)$ supercell along the paths passing through the high-symmetry points $\Gamma, \mathbf{X}, \mathbf{M}, \Gamma, \mathbf{X}^{\prime}, \mathbf{M}$. Panels (a) and (b) correspond to $\Omega_{0} \alpha / h=2 t$ and 20t, respectively, with $\alpha=0.2$ and $\gamma=0.8$. In the evolution from panel (a) to (b), the six central bands in panel (a), denoted with colors from orange to maroon, remain close in energy (as part of one single band, see perturbative analysis in [49]) while the remaining two bands in panel (a) (in cyan and brown) separate in energy and do not appear in panel (b).

the band structure for $E<0$ below. A six band manifold, close to the energy $-\Omega_{0}(1-\alpha)$ and well separated from nearest neighboring bands by energy $\Omega_{0} \alpha$, is shown along the high-symmetry points in Fig. 2 for (a) $\Omega_{0} \alpha / h=2 t$ and (b) $\Omega_{0} \alpha / h=20 t$. Within this six band manifold, the two middle bands closest to $-\Omega_{0}(1-\alpha)$ become quasiflat upon increasing $\Omega_{0} \alpha$, and are surrounded by a pair of dispersive Dirac cone spectra. The identification of quasiflatness follows from noticing that the dispersion of the middle bands (which is concentrated towards the edges of the Brillouin zone) is very small $\sim 0.2 t$ compared with the bandwidth of its immediately neighboring bands $(\sim 4 t)$ for $\Omega_{0} \alpha / h=20 t$. The flatness $F$ of these bands, calculated in perturbation theory, is proportional to $t /\left(4 \Omega_{0} \alpha\right)$ and equal to $\sim 0.02$ in the example above. Such values of $\Omega_{0}$ and $\alpha$ are feasible in our described experimental setup, leading to tunable bandwidth for the quasiflatbands. Figures 2(a) and 2(b) also show the associated density of states (DOS), which is given by $D(E)=L^{-d / 2} \sum_{i}\left[E-E\left(\mathbf{k}_{i}\right)\right]$.

Interestingly, band structures similar to the $\Theta(4,4)$ case appear when $l_{x}=l_{y}=4 \nu d$, with $\nu$ integer. This can be explained by treating the intralayer tunneling as a perturbation to the interlayer tunneling. As explained in detail in the Supplemental Material [49], the nodal lines of the periodic modulation determine a bilayer Lieb lattice of sites. The two layers are energetically well separated with on-site energies $\pm \Omega_{0}(1-\alpha)$, respectively. The perturbation then induces tunnelings within the Lieb lattice topology, which at first order are composed of nearest neighbor tunneling matrix elements within a single layer. The Lieb lattice in its simplest form [52] is known to host a pair of Dirac cones intersecting at a single $\mathbf{k}$ point on a completely flat band, the Dirac point. The dispersion of the flatbands in the full model described by Eq. (1) originates from higherorder contributions in perturbation theory. More generally, for $l_{x(y)}=4 \nu_{x(y)}$, where $\nu_{x(y)}$ are positive integers, a similar argument shows that the system can be effectively described by super-Lieb lattices with a supercell of $2\left(\nu_{x}+\nu_{y}\right)-1$ sites. Changing the periodicity of the Raman coupling in general leads to band structures without the above combination of Dirac spectra and flatbands [49].

An additional control parameter in our system is the artificial magnetic flux $\gamma$. It affects the band structure in a number of ways. As earlier, we focus on the case $\Theta_{M}=$ $\Theta(4,4)$ and the six bands closest to $E / t=-\Omega_{0}(1-\alpha)$. Increasing $\gamma$ leads to strong band narrowing. More interestingly, a nonzero $\gamma$ opens a local gap between the two quasiflatbands at the $\Gamma=(0,0)$ point in the Brillouin zone. Moreover, the lower Dirac cone detaches from the lower quasiflatband. This is reminiscent of the effect of a staggered chemical potential in the Lieb lattice [53]. The upper quasiflatband remains pinned exactly at the central energy $\left[E / t=-\Omega_{0}(1-\alpha)\right]$ around the $\Gamma$ point, and the upper Dirac cone remains gapless. Typical band configurations for two values of $\gamma$ are shown in Fig. 3. Interestingly, the flux $\gamma$ also controls the Dirac velocity of the cone which decreases with increasing flux. This result can be easily obtained from the perturbative mapping to the Lieb lattice [49] where, for small to intermediate values of $\gamma$, the

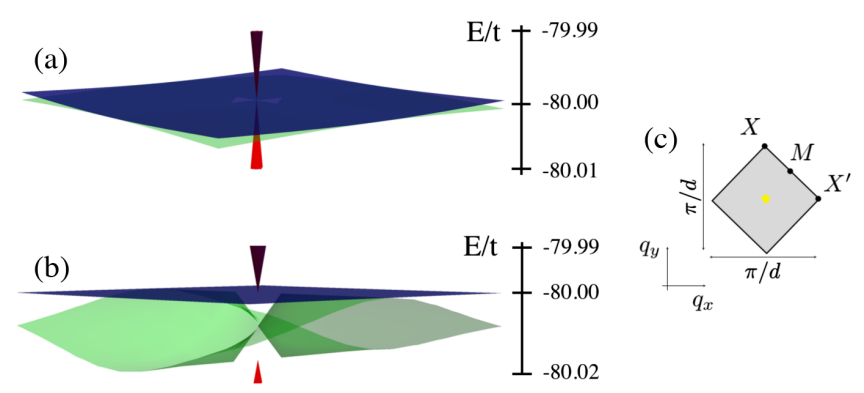

FIG. 3. Gap opening and Dirac cone widening due to the artificial flux $\gamma$. Four bands [Dirac cones (purple and red) and quasiflatbands (green and blue)] are shown in the vicinity of $E / t=-\Omega_{0}(1-\alpha)=-80$, for the $\Theta(4,4)$ system. The parameters and color scale of the bands are identical to those of Fig. 2(b), except for the magnetic flux that corresponds to (a) $\gamma=0$ and (b) $\gamma=0.8$. The energy surfaces are rotated for visibility, and only a small region centered at the $\Gamma=(0,0)$ point in the Brillouin zone [yellow area in (c), corresponding to $4 \%$ of the complete Brillouin zone] is depicted. 
dominant nearest neighbor tunneling (proportional to the Dirac velocity) is $\sim \cos (\gamma / 2)$. The Dirac velocity reduces to zero in the limit $\gamma \rightarrow \pi$ and the Dirac band becomes very narrow. Hence, both the quasiflatbands in between the Dirac spectra, as well as the Dirac spectra themselves can be controlled in our scheme.

Conclusions and outlook.-The basic element in the physics of TWBLG is the creation of large unit cells by rotating two layers with respect to each other. Around the magic angles, small rotations have a dramatic effect on the band structure of these systems. In this Letter, we have discussed a versatile method to create a new class of systems with controllable supercell structures for cold Fermi gases trapped in optical lattices. The size of the supercells is easily tunable and should allow addressing whether the physics of TWBLG is uniquely related to their macroscopic periodicity or, indeed, can be accessed for small unit cells. An inherent advantage of our opticallattice-based construction is the possibility to modify, over a wide range, the interlayer coupling, which is controlled by a combination of optical Raman transitions and excitedstate light shifts. We have shown that a square lattice synthetic bilayer displays a band structure that can be easily engineered by modifying the spatial periodicity, strength and Peierls phase imparted by the Raman lasers. As a result, magic periodicities of the interlayer coupling lead to the emergent quasiflatband physics.

The existence of identical scattering lengths parametrizing interactions between the atoms in the four internal states allows simulating the effect of both intralayer and interlayer interactions in the synthetic bilayer structure. The interacting Hamiltonian can be written as $H_{\mathrm{I}}=U / 2 \sum_{\mathbf{r}} n(\mathbf{r})[n(\mathbf{r})-1]$ where $n(\mathbf{r})=\sum_{m, \sigma} a_{m \sigma}^{\dagger}(\mathbf{r})$ $a_{m \sigma}(\mathbf{r})$, is the occupation of site (r) of the square optical lattice. The magnitude of $U$ could be tuned by varying the transverse confinement. In particular, choosing a value of $U$ smaller than $\Omega_{0} \alpha$ but much larger than the bandwidth of the quasiflatband should allow achieving the strongly interacting regime in the latter. Projection of interactions onto the quasiflat and hybridizing bands leads to extended Hubbard models with large on-site interactions as well as other terms, such as correlated tunneling. In this respect, it could be advantageous to open a hard gap between the quasiflatbands and neighboring dispersive Dirac bands. This can be done via additional mechanisms, such as lattice dimerization or spin-orbit coupling [54]. Probing such interacting systems at partial filling could potentially shed new light into theoretical debates on strongly correlated phenomena in twisted materials, such as unconventional superconductivity $[17,18,20,55]$ and topological order [21,56,57]. Finally, extending our approach to other lattice structures represents an exciting perspective for future studies.

We acknowledge funding from European Union (Grant No. ERC AdG NOQIA-833801), Ministerio de Ciencia, Innovación y Universidades (Grants No. FISICATEAMO
FIS2016-79508-P, No. QIBEQI FIS2016-80773-P, No. FIS2017-86530-P, No. QuDROP FIS2017-88334-P, and No. Severo Ochoa SEV-2015-0522), Deutsche Forschungsgemeinschaft (DFG, German Research Foundation) under Project No. 277974659 via Research Unit FOR 2414, Polish National Science Centre (Symfonia Grant No. 2016/20/W/ST4/00314), Generalitat de Catalunya (Grants No. SGR1341, No. SGR1381, No. SGR1646, No. SGR1660, and CERCA program), Fundació Privada Cellex, Fundació Mir-Puig, Fundación Ramón Areces, and European Social Fund. We acknowledge financial support from Secretaria d'Universitats i Recerca del Departament d'Empresa i Coneixement de la Generalitat de Catalunya, co-funded by the European Union Regional Development Fund within the ERDF Operational Program of Catalunya (project QuantumCat, ref. 001-P-001644). A. C. acknowledges additional support from the Talent Research program of the Universitat Autònoma de Barcelona, T.S. from the Secretaria d'Universitats i Recerca de la Generalitat de Catalunya and the European Social Fund, R. W. C. from the Polish National Science Centre $(\mathrm{NCN})$ under Maestro Grant No. DEC-2019/34/A/ST2/00081, L. T. from Ministerio de Ciencia, Innovación y Universidades (Grant No. RYC-2015-17890), and I.F. and D. R. from the Fundació Cellex through Cellex-ICFO-MPQ.

[1] A. Geim and I. Grigorieva, Van der Waals heterostructures, Nature (London) 499, 419 (2013).

[2] Y. Cao, V. Fatemi, S. Fang, K. Watanabe, T. Taniguchi, E. Kaxiras, and P. Jarillo-Herrero, Unconventional superconductivity in magic-angle graphene superlattices, Nature (London) 556, 43 (2018).

[3] Y. Cao, V. Fatemi, A. Demir, S. Fang, S. L. Tomarken, J. Y. Luo, J. D. Sanchez-Yamagishi, K. Watanabe, T. Taniguchi, E. Kaxiras, R. C. Ashoori, and P. Jarillo-Herrero, Correlated insulator behaviour at half-filling in magic-angle graphene superlattices, Nature (London) 556, 80 (2018).

[4] M. Yankowitz, S. Chen, H. Polshyn, Y. Zhang, K. Watanabe, T. Taniguchi, D. Graf, A. F. Young, and C. R. Dean, Tuning superconductivity in twisted bilayer graphene, Science 363, 1059 (2019).

[5] X. Lu, P. Stepanov, W. Yang, M. Xie, M. A. Aamir, I. Das, C. Urgell, K. Watanabe, T. Taniguchi, G. Zhang, A. Bachtold, A. H. MacDonald, and D. K. Efetov, Superconductors, orbital magnets, and correlated states in magic angle bilayer graphene, Nature (London) 574, 653 (2019).

[6] G. E. Volovik, Graphite, graphene, and the flat band superconductivity, JETP Lett. 107, 516 (2018).

[7] N. F. Q. Yuan and L. Fu, Model for the metal-insulator transition in graphene superlattices and beyond, Phys. Rev. B 98, 045103 (2018).

[8] M. Koshino, N. F. Q. Yuan, T. Koretsune, M. Ochi, K. Kuroki, and L. Fu, Maximally Localized Wannier Orbitals and the Extended Hubbard Model for Twisted Bilayer Graphene, Phys. Rev. X 8, 031087 (2018). 
[9] M. Ochi, M. Koshino, and K. Kuroki, Possible correlated insulating states in magic-angle twisted bilayer graphene under strongly competing interactions, Phys. Rev. B 98, 081102(R) (2018).

[10] L. Zou, H. C. Po, A. Vishwanath, and T. Senthil, Band structure of twisted bilayer graphene: Emergent symmetries, commensurate approximants, and Wannier obstructions, Phys. Rev. B 98, 085435 (2018).

[11] T. J. Peltonen, R. Ojajärvi, and T. T. Heikkilä, Mean-field theory for superconductivity in twisted bilayer graphene, Phys. Rev. B 98, 220504 (2018).

[12] B. Padhi, C. Setty, and P. W. Phillips, Doped twisted bilayer graphene near magic angles: Proximity to Wigner crystallization not Mott insulation, Nano Lett. 18, 6175 (2018).

[13] A. O. Sboychakov, A. V. Rozhkov, A. L. Rakhmanov, and F. Nori, Many-body effects in twisted bilayer graphene at low twist angles, Phys. Rev. B 100, 045111 (2019).

[14] F. Guinea and N. R. Walet, Electrostatic effects, band distortions, and superconductivity in twisted graphene bilayers, Proc. Natl. Acad. Sci. U.S.A. 115, 13174 (2018).

[15] X. Y. Xu, K. T. Law, and P. A. Lee, Kekulé valence bond order in an extended Hubbard model on the honeycomb lattice with possible applications to twisted bilayer graphene, Phys. Rev. B 98, 121406 (2018).

[16] F. Wu, A. H. MacDonald, and I. Martin, Theory of PhononMediated Superconductivity in Twisted Bilayer Graphene, Phys. Rev. Lett. 121, 257001 (2018).

[17] H. Isobe, N. F. Q. Yuan, and L. Fu, Unconventional Superconductivity and Density Waves in Twisted Bilayer Graphene, Phys. Rev. X 8, 041041 (2018).

[18] Y.-Z. You and A. Vishwanath, Superconductivity from valley fluctuations and approximate $S O(4)$ symmetry in a weak coupling theory of twisted bilayer graphene, arXiv:1805.06867.

[19] B. Lian, Z. Wang, and B. A. Bernevig, Twisted Bilayer Graphene: A Phonon Driven Superconductor, Phys. Rev. Lett. 122, 257002 (2019).

[20] Y.-P. Lin and R. M. Nandkishore, A chiral twist on the highTc phase diagram in Moiré heterostructures, Phys. Rev. B 100, 085136 (2019).

[21] Z. Song, Z. Wang, W. Shi, G. Li, C. Fang, and B. A. Bernevig, All Magic Angles in Twisted Bilayer Graphene are Topological, Phys. Rev. Lett. 123, 036401 (2019).

[22] E. Suárez Morell, J. D. Correa, P. Vargas, M. Pacheco, and Z. Barticevic, Flat bands in slightly twisted bilayer graphene: Tight-binding calculations, Phys. Rev. B 82, 121407 (2010).

[23] R. Bistritzer and A. H. MacDonald, Moiré bands in twisted double-layer graphene, Proc. Natl. Acad. Sci. U.S.A. 108, 12233 (2011).

[24] J. M. B. Lopes dos Santos, N. M. R. Peres, and A. H. Castro Neto, Continuum model of the twisted graphene bilayer, Phys. Rev. B 86, 155449 (2012).

[25] P. Moon and M. Koshino, Energy spectrum and quantum Hall effect in twisted bilayer graphene, Phys. Rev. B 85, 195458 (2012).

[26] G. Tarnopolsky, A. J. Kruchkov, and A. Vishwanath, Origin of Magic Angles in Twisted Bilayer Graphene, Phys. Rev. Lett. 122, 106405 (2019).
[27] M. Polini, F. Guinea, M. Lewenstein, H. C. Manoharan, and V. Pellegrini, Artificial graphene as a tunable Dirac material, Nat. Nanotechnol. 8, 625 (2013).

[28] P. Wang, Y. Zheng, X. Chen, C. Huang, Y. V. Kartashov, L. Torner, V. V. Konotop, and F. Ye, Localization and delocalization of light in photonic moiré lattices, Nature (London) 577, 42 (2020).

[29] I. Bloch, J. Dalibard, and W. Zwerger, Many-body physics with ultracold gases, Rev. Mod. Phys. 80, 885 (2008).

[30] M. Lewenstein, A. Sanpera, and V Ahufinger, Ultracold Atoms in Optical Lattices: Simulating Quantum Many-Body Systems (Oxford University Press, Oxford, 2012).

[31] P. Soltan-Panahi, J. Struck, P. Hauke, A. Bick, W. Plenkers, G. Meineke, C. Becker, P. Windpassinger, M. Lewenstein, and K. Sengstock, Multi-component quantum gases in spindependent hexagonal lattices, Nat. Phys. 7, 434 (2011).

[32] L. Tarruell, D. Greif, T. Uehlinger, G. Jotzu, and T. Esslinger, Creating, moving and merging Dirac points with a Fermi gas in a tunable honeycomb lattice, Nature (London) 483, 302 (2012).

[33] G.-B. Jo, J. Guzman, C. K. Thomas, P. Hosur, A. Vishwanath, and D. M. Stamper-Kurn, Ultracold Atoms in a Tunable Optical Kagome Lattice, Phys. Rev. Lett. 108, 045305 (2012).

[34] S. Taie, H. Ozawa, T. Ichinose, T. Nishio, S. Nakajima, and Y. Takahashi, Coherent driving and freezing of bosonic matter wave in an optical Lieb lattice, Sci. Adv. 1, e1500854 (2015).

[35] H. Ozawa, S. Taie, T. Ichinose, and Y. Takahashi, Interaction-Driven Shift and Distortion of a Flat Band in an Optical Lieb Lattice, Phys. Rev. Lett. 118, 175301 (2017).

[36] L. Guidoni, C. Triché, P. Verkerk, and G. Grynberg, Quasiperiodic Optical Lattices, Phys. Rev. Lett. 79, 3363 (1997).

[37] K. Viebahn, M. Sbroscia, E. Carter, Jr-C. Yu, and U. Schneider, Matter-Wave Diffraction from a Quasicrystalline Optical lattice, Phys. Rev. Lett. 122, 110404 (2019).

[38] S. V. Rajagopal, T. Shimasaki, P. Dotti, M. Raciũnas, R. Senaratne, E. Anisimovas, A. Eckardt, and D. M. Weld, Phasonic Spectroscopy of a Quantum Gas in a Quasicrystalline lattice, Phys. Rev. Lett. 123, 223201 (2019).

[39] A. González-Tudela and J. I. Cirac, Cold atoms in twisted bilayer optical potentials, Phys. Rev. A 100, 053604 (2019).

[40] T. Graß, R. W. Chhajlany, L. Tarruell, V. Pellegrini, and M. Lewenstein, Proximity effects in cold atom artificial graphene, 2D Mater. 4, 015039 (2016).

[41] O. Boada, A. Celi, J. I. Latorre, and M. Lewenstein, Quantum Simulation of an Extra Dimension, Phys. Rev. Lett. 108, 133001 (2012).

[42] A. Celi, P. Massignan, J. Ruseckas, N. Goldman, I. B. Spielman, G. Juzeliunas, and M. Lewenstein, Synthetic Gauge Fields in Synthetic Dimensions, Phys. Rev. Lett. 112, 043001 (2014).

[43] T. Ozawa and H. M. Price, Topological quantum matter in synthetic dimensions, Nat. Rev. Phys. 1, 349 (2019).

[44] To suppress optical transitions to other spin states, an additional laser beam of smaller detuning and producing a large differential light shift can be used [45]. 
[45] S. Stellmer, R. Grimm, and F. Schreck, Detection and manipulation of nuclear spin states in fermionic strontium, Phys. Rev. A 84, 043611 (2011).

[46] S. Stellmer, B. Pasquiou, R. Grimm, and F. Schreck, Laser Cooling to Quantum Degeneracy, Phys. Rev. Lett. 110, 263003 (2013).

[47] C.-C. Chen, S. Bennetts, R. González Escudero, F. Schreck, and B. Pasquiou, Sisyphus optical lattice decelerator, Phys. Rev. A 100, 023401 (2019).

[48] In this regime, the interlayer tunneling remains smaller than the band gap between the first and second band of the realspace lattice $\Delta E / h=13.5 \mathrm{kHz}$.

[49] See Supplemental Material at http://link.aps.org/ supplemental/10.1103/PhysRevLett.125.030504 for the derivation of the effective models, which includes Refs. [50,51].

[50] A. Kerelsky, L. McGilly, D. M. Kennes, L. Xian, M. Yankowitz, S. Chen, K. Watanabe, T. Taniguchi, J. Hone, C. Dean, A. Rubio, and A. N. Pasupathy, Magic angle spectroscopy, Nature (London) 572, 95 (2019).
[51] N. F. Q. Yuan, H. Isobe, and L. Fu, Magic of high-order van Hove singularity, Nat. Commun. 10, 5769 (2019).

[52] E. H. Lieb, Two Theorems on the Hubbard Model, Phys. Rev. Lett. 62, 1201 (1989).

[53] R. Shen, L. B. Shao, Baigeng Wang, and D. Y. Xing, Single Dirac cone with a flatband touching on line-centered-square optical lattices, Phys. Rev. B 81, 041410(R) (2010).

[54] T. Salamon et al. (unpublished).

[55] J. González and T. Stauber, Kohn-Luttinger Superconductivity in Twisted Bilayer Graphene, Phys. Rev. Lett. 122, 026801 (2019).

[56] M. J. Park, Y. Kim, G. Y. Cho, and S. Lee, Higher-Order Topological Insulator in Twisted Bilayer Graphene, Phys. Rev. Lett. 123, 216803 (2019).

[57] C. Ma, Q. Wang, S. Mills, X. Chen, B. Deng, S. Yuan, C. Li, K. Watanabe, T. Taniguchi, D. Xu, F. Zhang, and F. Xia, Discovery of high dimensional band topology in twisted bilayer graphene, arXiv:1903.07950. 